\title{
Residual Stress Measurement of Additively Repaired Ti-6Al-4V Using Fibre Optic Sensing
}

\author{
Matthew Stevens ${ }^{1, \mathrm{a}}{ }^{*}$, Kieran Pryor ${ }^{2, \mathrm{~b}}$ and Suzana Turk ${ }^{3, \mathrm{c}}$ \\ ${ }^{1}$ Defence Science and Technology Group, 506 Lorimer Street, Fishermans Bend, VIC, 3207, \\ Australia \\ amatthew.stevens@dst.defence.gov.au, bkieran.pryor@dst.defence.gov.au, \\ csuzana.turk@dst.defence.gov.au \\ (C) 2020 Commonwealth of Australia
}

Keywords: Fibre Optics, Distributed Strain Sensing, Continuous Fibre Gratings, Residual Stress, Laser Metal Deposition

\begin{abstract}
Laser metal deposition (LMD) is a laser-based additive manufacturing (AM) technology that offers significant advantages in the production and repair of bespoke and valuable parts targeting applications in the aerospace, tooling and medical industries. A significant problem with AM is the development of high residual stresses, deformation and cracking. Advanced sensing technologies can be a useful tool for characterising residual stress (RS) and the structural response of AM aerospace components under fatigue loading conditions. This paper reports on a feasibility study assessing the performance of fibre optic (FO) distributed strain measurement technology to measure surface RS in comparison to traditional electrical resistance strain gauges and the contour method. The results from this study will be used to justify further experimental work.
\end{abstract}

\section{Introduction}

LMD applications have been targeted for repairing intricate geometries and structural restoration of specialised high value components such as sintered tools and aerospace components [1,2]. Metallic powder is blown through a deposition nozzle and heated with a laser to produce a metallic bead that is deposited, layer by layer and track by track, to build-up a part or to add layers of material to an existing part. The nozzle system shrouds the work area with shield gas thereby eliminating the requirement for enclosures with controlled environments and thus providing exciting opportunities for Defence applications.

LMD delivers high density deposited material with a strong metallurgical bond to the substrate. However, the repeated and highly localised heating and cooling lead to non-uniform thermal expansion and contraction which results in a complicated distribution of RS in the heat affected zone (HAZ) and distortion of the part [3,4]. Similar to the HAZ in welding, RSs are detrimental to the structural integrity of the part, with warping and stress concentrations promoting fatigue cracking and inducing unpredictable buckling in service.

The nature and magnitude of RS in the deposited material and substrate affect the integrity of the component. In a previous study RS was reported to be lower transverse to the deposition track and for shorter track lengths, due to higher remnant heat leading to smaller temperature gradients which in turn leads to lower RS [5]. The HAZ and molten pool had an elongated shape in the deposition direction and the temperature gradient was pronounced due to the relatively low thermal conductivity of Ti6Al4V [6]. This could induce an uneven RS profile with peak RS coinciding with the final track deposit and laser stop position [7]. 
Methods for measuring RS include the contour method, a destructive and time-intensive test that determines RS by creating a free surface and measuring the resulting deformation due to RS redistribution. It delivers a $2 \mathrm{D}$ through-thickness RS contour map. Neutron diffraction delivers a non-destructive through-thickness line measurement but is sample-size restrictive and timeintensive. In the present study, a non-destructive alternative is proposed using FO sensing (FOS), a rapidly evolving technology which has been considered for structural health monitoring of airframes, bridges and pipelines.

Developments in FOS using continuous fibre gratings allow this technology to offer a densely distributed strain measurement capability. Distributed sensing is achieved using a low reflectivity grating, which is a small periodic change in refractive index of the glass fibre core, written over the entire fibre length as it is being fabricated in the draw tower. This configuration is known as a continuous fibre grating [8]. These sensing systems rely on spatially interrogating the backscattered light from the gratings via optical frequency domain reflectometry (OFDR), which measures the spectral shift of the backscattered light in time (via a fast Fourier transform) and then scales this shift by the known number of gratings to correlate changes in length to corresponding changes in strain [9].

It was proposed that FOS could potentially deliver surface RS measurements comparable to the well-established contour method in a shorter timeframe. Therefore, this paper reports on a feasibility study assessing the performance of FO distributed strain measurement technology to measure surface RS in comparison to industry standard electrical resistance foil strain gauges (FSGs) and the contour method reported in [4]. The results from this feasibility study will be used to justify further experimental work.

\section{Experimental Method}

Material. Rectangular section fatigue coupons, measuring $200 \times 19 \times 6.35 \mathrm{~mm}$, were manufactured from Ti-6Al-4V plate, Figure 1(a). A section measuring $19 \times 12.7 \times 0.8 \mathrm{~mm}$ was machined out of the centre gauge length (Figure 1, BL-GO) and repaired with one layer of laser deposited Ti-6Al-4V powder. The RS distributions of two deposition strategies were studied: LMD-1 is a continuous raster scan with deposition tracks parallel to the coupon length and loading direction, Figure 1(b); and LMD-2 which is a continuous raster scan with deposition tracks perpendicular to the coupon length and loading direction, Figure 1(c). Five coupons were tested in total, two LMD-1 and three LMD-2 type coupons. The baseline and baseline grind-out coupons are shown in Figure 1(a) for reference only. Thermal cycling and RS from the repair process resulted in coupon bowing, Figure 2.

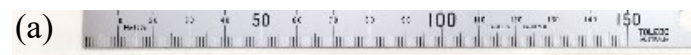

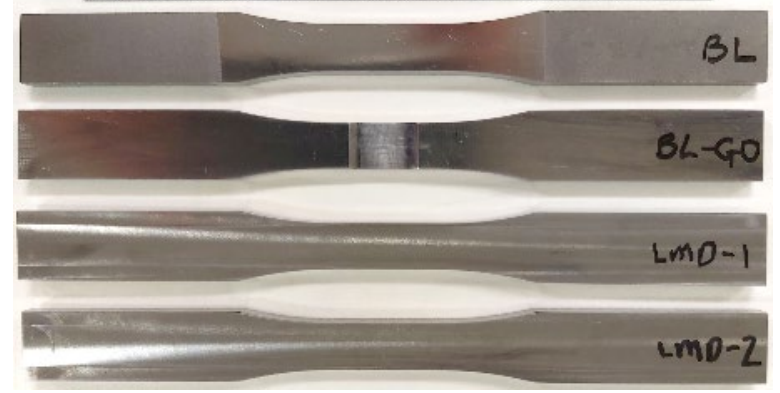

(b)

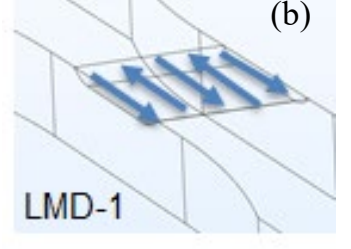

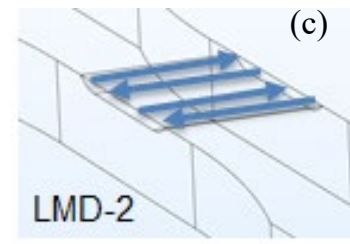

(c)

Figure 1. Fatigue coupons: (a) baseline (BL) with no repair; baseline grind-out (BL-GO) with no repair but indicates repair area; laser metal deposition (LMD-1) repair tracks parallel to coupon gauge length and loading direction, refer to (b); and (LMD-2) repair tracks perpendicular to coupon gauge length and loading direction, refer to (c). 


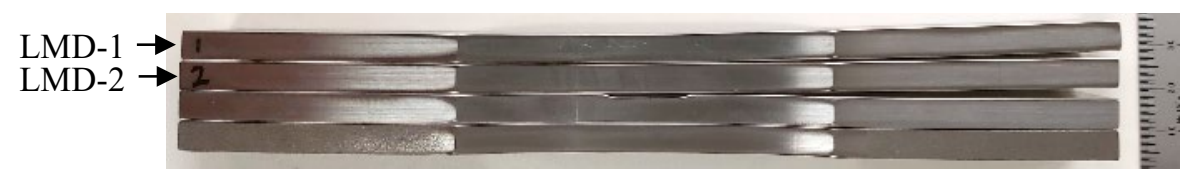

Figure 2. Side view of fatigue coupons pinned down on left hand side, showing BL (bottom), $B L$ $G O$ (centre) and bowing of LMD-1 and LMD-2 repaired coupons (top, labelled LMD-1 and

$L M D-2)$.

Instrumentation. The coupons were instrumented with a $3 \mathrm{~m}$ sensing length of commercially supplied all grating fibre (FBGS) that was interrogated using an OFDR based measurement system (Sensuron Summit). This sensing system combination offers a minimum spatial resolution of $1.6 \mathrm{~mm}$ and a notional $\pm 1 \mu \varepsilon$ accuracy.

Loctite EA9309.3NA epoxy was used to adhere the sensing fibres to the titanium alloy substrate in parallel lines along the gauge and loading axis in a looping format with unbonded loops, Figure 3(a). Twelve sensing lines were adhered with four parallel lines on the repair face and back face and two parallel lines on the side gauges, Figure 3(b).
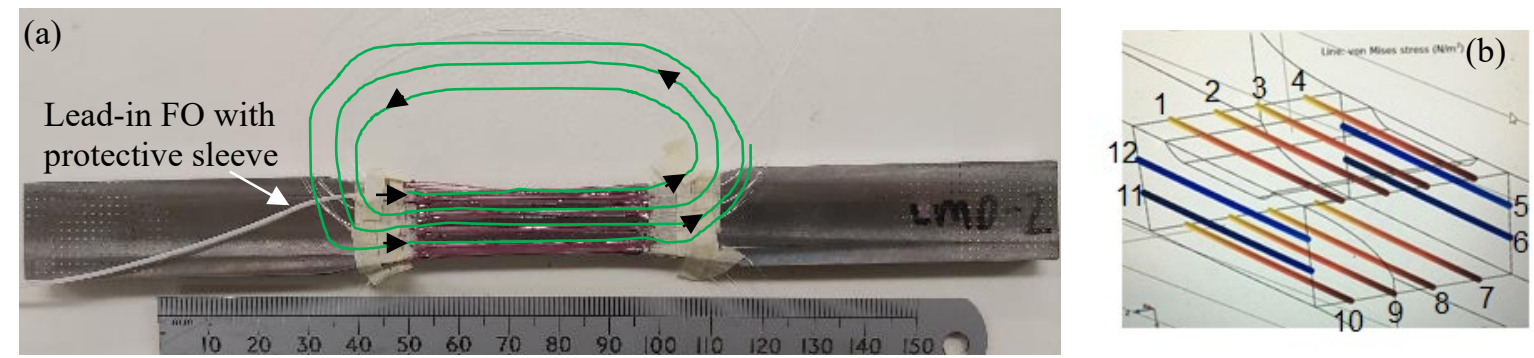

Figure 3. FO instrumentation layout: (a) lead-in FO begins at top left with four parallel lines adhered on the repair face showing unbonded loops, and (b) adhering and looping format continued wrapping around the coupon in a clockwise direction.

An example LMD-2 coupon was instrumented with FOS and FSGs for direct strain data comparison and validation with an industry standard technique. Two Kyowa copper plated 120ohm KFG-1-120-D9-11N10C2 FSGs were adhered in line along one half of the coupon gauge length and repair area to measure strain in the direction of loading, Figure 4. An optical fibre was adhered in line with the FSGs on the other half of the coupon, with another two FO lines adhered directly adjacent to the FSGs and spanning the gauge length. The strain gauges were adhered to the titanium alloy substrate using M-Bond adhesive. These FSGs provide $2 \mathrm{~mm}$ spatial resolution and a notional $\pm 1 \mu \varepsilon$ accuracy. A National Instruments Data Acquisition (NI DAQ) chassis and NI-9235 8-channel C series strain/bridge input module were used to capture FSG data. For comparison to published data, FOS and FSG strain data were converted to stress using Equation 1 , where $\sigma$ is stress, $\mathrm{E}$ is elastic modulus $(121 \mathrm{GPa})$ and $\varepsilon$ is the measured strain.

$$
\sigma=\mathrm{E} \times \varepsilon
$$

Load Application. Coupon loads were applied using a $100 \mathrm{kN}$ servo-hydraulic mechanical testing machine in a National Association of Testing Authorities (NATA) certified laboratory. The upper coupon grip was secured with hydraulic wedge-grip jaw faces. While the lower coupon grip was unclamped, instrumentation was calibrated and zeroed. Residual stress was recorded when the lower coupon grip was secured with grip pressure and zero applied load, 
forcing the bowed coupon to straighten. Therefore, the RS distributions reported in this paper were equal in magnitude but opposite in sign to those accumulated during the repair, that is, RS was measured in reverse. FSG and FO strain data was recorded during a 0-40 $\mathrm{kN}$ ramp load excursion to enable comparison between the two sets of measurements.

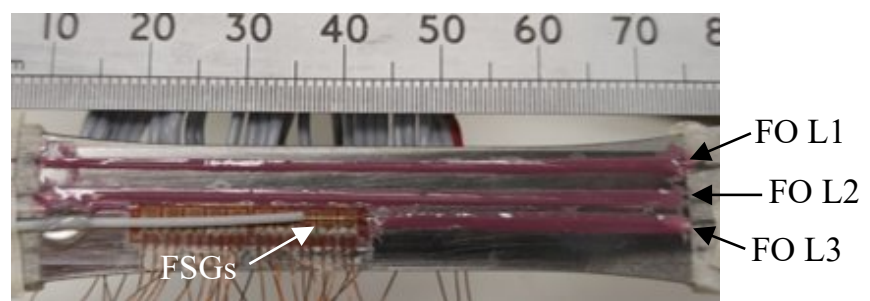

Figure 4. Two FSGs (labelled) were instrumented in line along one half of the coupon gauge length and repair area to measure strain in the direction of loading. An FO sensor was adhered in line with the FSGs on the other half of the coupon (labelled FO L3) with a second (labelled FO L2) and third FO line (labelled FO L3) adhered directly adjacent to the FSGs and spanning the gauge length.

\section{Results and Discussion}

Strain profiles along the gauge length measured with the FOS and FSGs at zero uniaxial load and at $40 \mathrm{kN}$ are compared in Figure 5, with the data arranged as instrumented in Figure 4. It is to be noted that when the coupon is clamped under grip pressure a straightening moment results, which explains the non-zero RS profile at zero applied load. The strain profiles are in general agreement, with evidence of an uneven RS profile both along and across the gauge length. This observation is consistent with other reported studies [4,6]. The highest RS was recorded in FO L2, with a peak value of $153 \mathrm{MPa}$, while the peak stress value at maximum applied load occurred in FO L1. Although not shown here, coupon fatigue initiation and fracture occurred between FO L1 and FO L2 approximately $-6 \mathrm{~mm}$ from the repair centre where the FO line graphs overlap at maximum applied load, Figure 5(b). The peak RS for the five coupons tested are summarised in Table 1 and ranged between 107-179 $\mathrm{MPa}$, with these values correlating well with the severity of bowing. This range is consistent with near-surface RS values measured by the contour method reported in [4]. RS values are expected to vary between coupons due to thermal cycling of multiple clad coupons on a build plate which leads to different bowing profiles. These results show that FOS can provide a reasonable estimate for the RS distribution and of the peak stress at high load, for this application.

Table 1. Summary of measured coupon bowing and residual stress obtained from FO sensors.

\begin{tabular}{|c|c|c|c|}
\hline $\begin{array}{c}\text { Repair } \\
\text { Strategy }\end{array}$ & $\begin{array}{c}\text { Bowing } \\
{[\mathrm{mm}]}\end{array}$ & $\begin{array}{c}\text { Peak Residual } \\
\text { Strain }[\mu \varepsilon]\end{array}$ & $\begin{array}{c}\text { Peak Residual } \\
\text { Stress [MPa] }\end{array}$ \\
\hline LMD-1 & 5.06 & 1478 & 179 \\
\hline LMD-1 & 3.74 & 1047 & 127 \\
\hline LMD-2 & 3.05 & 882 & 107 \\
\hline LMD-2 & 4.21 & 1220 & 148 \\
\hline LMD-2 & 4.77 & 1265 & 153 \\
\hline
\end{tabular}



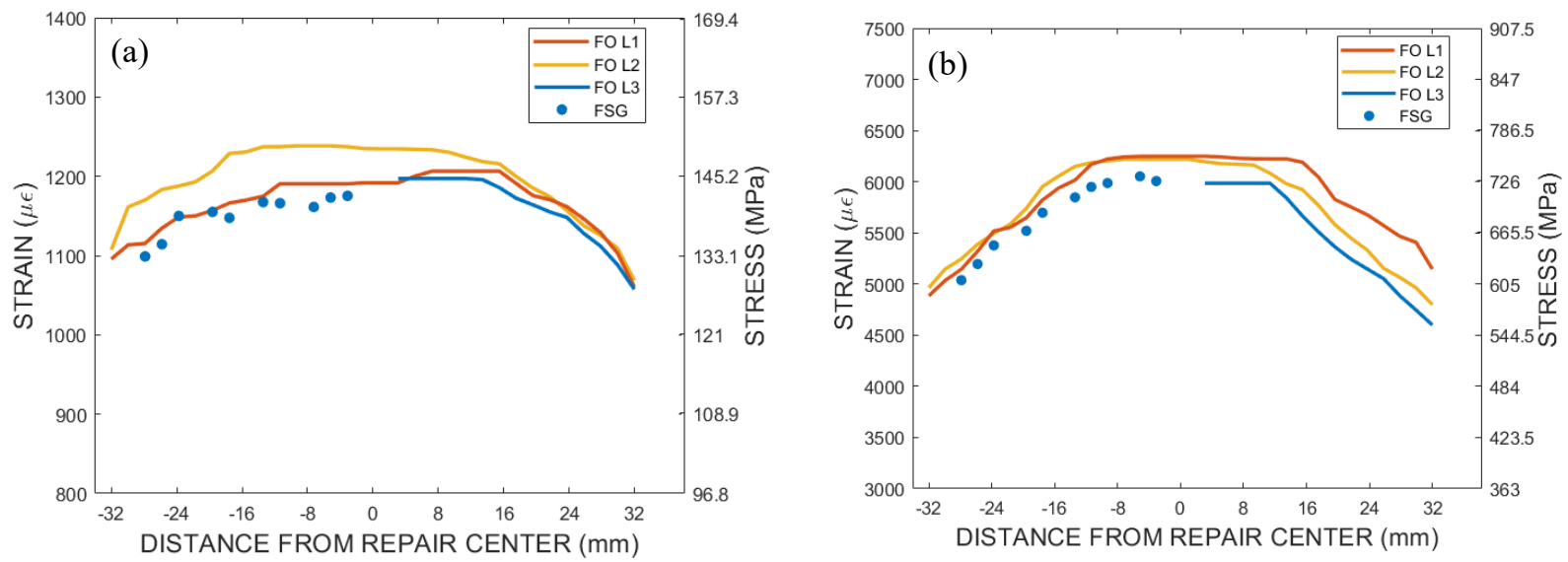

Figure 5. Example LMD-2 strain profiles along the gauge length from FO sensors and FSGs are presented at two load conditions: zero applied load, corresponding to residual stress distribution (a), and at $40 \mathrm{kN}$, corresponding to an applied stress of $500 \mathrm{MPa}(\mathrm{b})$.

Results from the coupon fatigue testing, including FOS determined RS values and profiles, are presented in the following format through Figures 6-9: the residual stress distribution along the entire fibre length for all four coupon sides (refer to Figure 3), a magnified view of the peak RS which is shown in the same orientation as the coupon photos, i.e. left to right along the gauge length, and coupon photos showing the instrumented gauge, the machined section indicating repair area and the fatigue fracture location.

LMD-1 Results. The RS distribution for an example LMD-1 coupon is shown in Figure 6(a). As mentioned previously, RS values reported in this paper are equal in magnitude but opposite in sign to those accumulated during the repair. Under grip pressure and zero axial load, the repair face was in tension while the back face was in compression. Regions in the graphs showing zero strain correspond to un-bonded fibre, i.e. the looped fibre at the end of each adhered line, Figure 6(a) and 6(b). The RS profile both along and across the gauge length was uneven, indicated respectively by the different peak values and asymmetric profiles labelled F1 to F4 in Figure 6(a) (the corresponding FOS lines are labelled in Figure 6(c)). These profiles indicate increasing RS along the gauge length from left to right and across the gauge from F4 to F1. The maximum RS, corresponding to profile F1 (shown magnified in Figure 6b), was $179 \mathrm{MPa}$ and occurred at the repair and HAZ interface. This maximum coincided with the coupon fracture and fatigue initiation location (arrowed in Figure 6c).

The RS distribution for another example LMD-1 coupon is shown in Figure 7(a). The RS profile both along and across the gauge length was uneven, with profiles labelled F1 to F4 showing increasing RS along the gauge length from left to right and across the gauge from F1 to F4. The maximum RS corresponding to profile F4 was $127 \mathrm{MPa}$ and coincided with the coupon fracture and fatigue initiation location (arrowed in Figure 7c). 
(a)

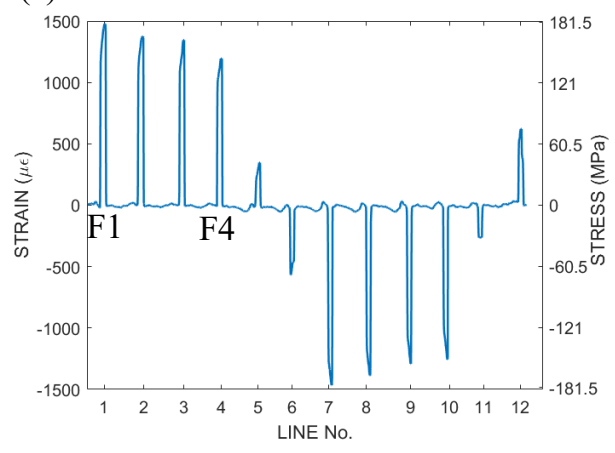

(b)

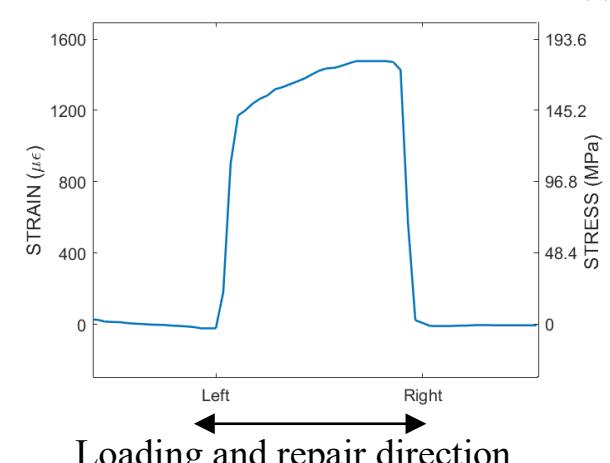

(c)

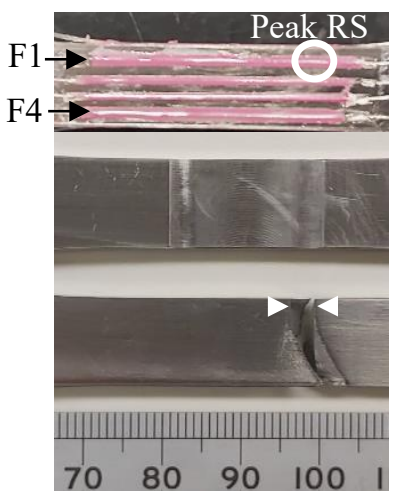

Figure 6. The RS profile labelled F1, shown magnified in (b) and labelled in (c), indicates the peak $R S$ location. Peak RS aligned with the fatigue crack initiation and fracture location arrowed in (c).

(a)

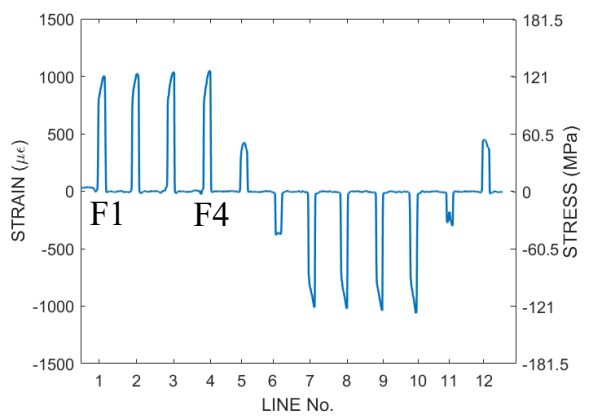

(b)

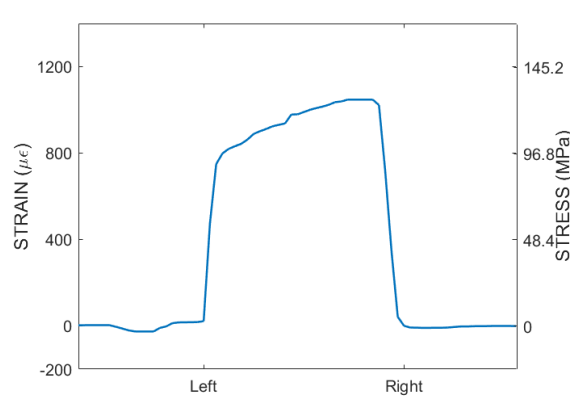

(c)

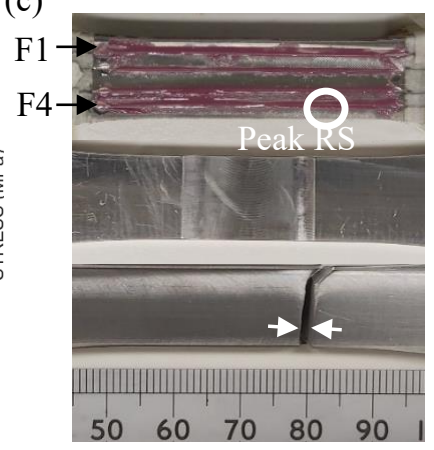

Figure 7. As in Figure 6, the RS distribution for another representative LMD-1 coupon. Peak RS aligned with the fatigue crack initiation and fracture location arrowed in (c).

LMD-2 Results. The RS distribution for an example LMD-2 coupon is shown in Figure 8(a), noting FOS instrumentation began on the back of the coupon in this case. The RS profile both along and across the gauge length was more evenly distributed than for the LMD-1 coupons, referring to profiles labelled F7 to F10 in Figure 8(a) and 8(c). The peak RS occurred in F10 (shown magnified in Figure $8 \mathrm{~b}$ and labelled in Figure $8 \mathrm{c}$ ) and was $\sim 148 \mathrm{MPa}$. This peak RS coincided with the coupon fracture and fatigue initiation location (arrowed in Figure 8c).

(a)

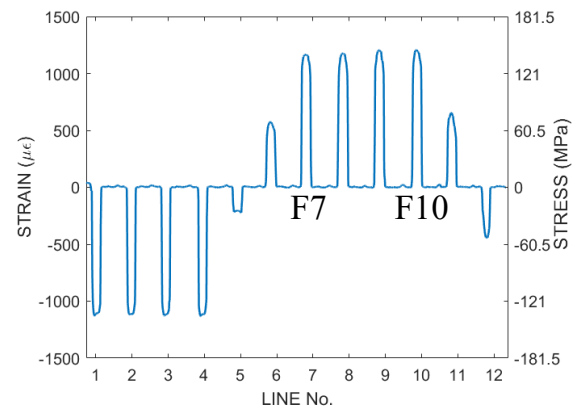

(b)

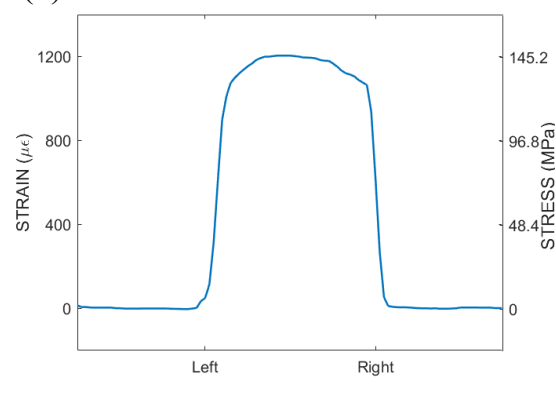

(c)

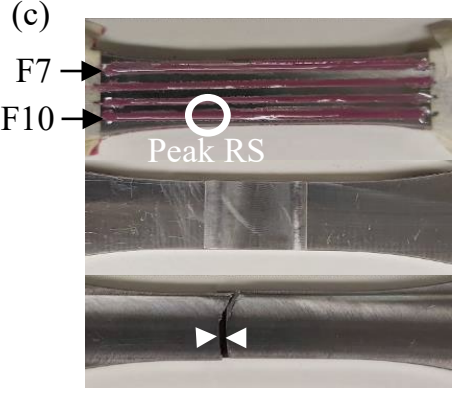

Figure 8. The RS distribution for a representative LMD-2 coupon. Peak RS aligned with the fatigue crack initiation and fracture location arrowed in (c). 
The RS distribution for another example LMD-2 coupon is shown in Figure 9(a). The RS profile along the gauge length was uneven, referring to the four asymmetric profiles labelled F1 to F4. These profiles showed increasing RS along the gauge length from left to right. The maximum RS corresponding to profile F3 was $107 \mathrm{MPa}$ and did not coincide with coupon fracture and fatigue initiation location (arrowed in Figure 9c). In this case, fatigue cracking initiated and propagated from the coupon side and although significant RS was noted on both coupon sides it did not align with the fracture and initiation location. A significant surfacebreaking feature was observed at the fatigue crack origin outside of the repair area in the substrate material. The stress concentration associated with the feature likely had a greater impact on fatigue crack initiation than RS.

(a)

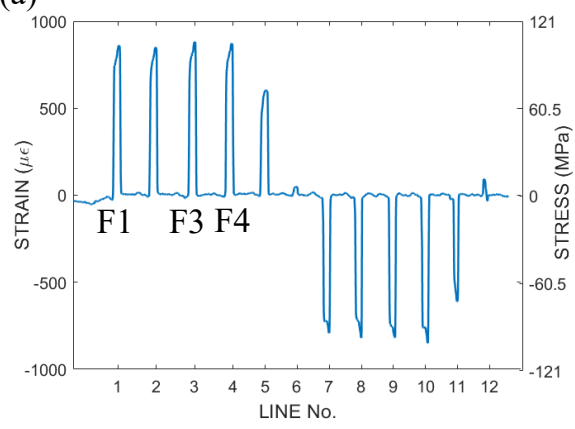

(b)

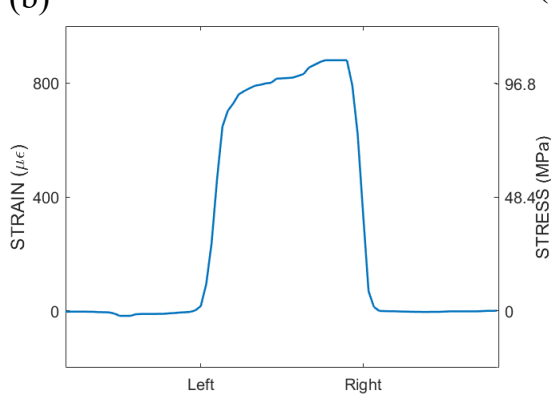

(c)

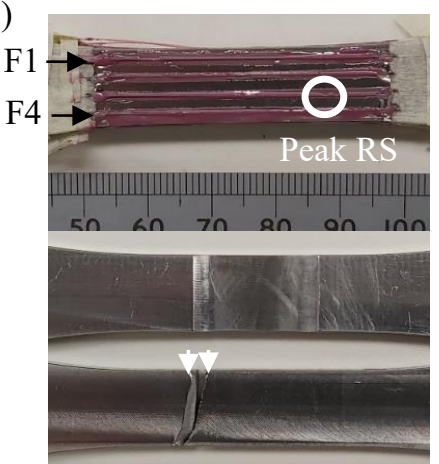

Figure 9. RS distribution for another LMD-2 coupon is shown in (a). Peak RS did not align with the fatigue crack initiation and fracture location, arrowed in (c).

Overall, the LMD-1 strategy produced a significantly uneven RS profile both across and along the gauge length in the deposition direction compared to LMD-2. This was consistent with the HAZ and molten pool having an elongated shape in the deposition direction and it is likely that the peak RS coincided with the final track deposit and laser stop position [6,7]. LMD-2 produced a more evenly distributed RS profile with a slight trend towards the laser stop position. This is likely due to the shorter deposition tracks having higher remnant heat which leads to smaller temperature gradients and lower RS.

\section{Conclusions}

RS values derived from FOS and FSGs were generally consistent, with the peak RS for the five coupons tested ranging between 107-179 $\mathrm{MPa}$. This range was also consistent with near-surface RS values determined by the contour method. RS values and profiles are expected to vary between coupons due to thermal cycling of multiple clad coupons on a build plate. The results showed FOS strain measurements to reflect with relatively good accuracy the surface RS distribution and peak stress values under load, thereby validating the suitability of FOS for the investigation of surface RS in laser repaired coupons of the type considered. Additionally, the study showed FOS can identify surface RS hotspots and therefore potential failure locations. Due to the limited number of tests in the present study, further testing is required to confirm this correlation and understand the effect of applied stress on fatigue initiation and fracture location, as well as fatigue life.

The FOS RS estimates reported in this paper were obtained by forcing bowed coupons to straighten, which limits the application of this approach to relatively thin structures. Although, the contour method provides a through-thickness RS map, it is destructive and cannot be economically applied for every LMD sequence. However, with FOS used as a complimentary 
technique, it was possible to characterise surface RS for every coupon in the test matrix which identified correlations between peak RS values and the failure location.

Future work will investigate the RS distribution and structural response of LMD repairs during cyclic loading. The test matrix will incorporate two stress levels and repair strategies to establish a correlation between repair direction, fatigue life and stress with fatigue fracture initiation and location. The aim is to understand whether RS mapping can assist prediction of failure location of repaired coupons. These findings will be validated with post-test fractography.

\section{Acknowledgements}

The authors gratefully acknowledge Ms Yi Rye Choi for providing her coupons as a platform to demonstrate the fibre optic sensing capability. They also acknowledge the facilities and technical assistance of the Fatigue and Fracture Laboratory, Mr Daniel Bitton and Material State Awareness Laboratory at DST Group.

\section{References}

[1] Q. Liu, M. Janardhana, B. Hinton, M. Brandt \& K. Sharp, Laser cladding as a potential repair technology for damaged aircraft components. International Journal of Structural Integrity (2011) 2:314-31. https://doi.org/10.1108/17579861111162914

[2] M. Leino, J. Pekkarinen \& R. Soukka, The role of laser additive manufacturing methods of metals in repair, refurbishment and remanufacturing - enabling circular economy. Physics Proceedings (2016) 83:752-60. https://doi.org/10.1016/j.phpro.2016.08.077

[3] H. Liu \& F. Liou, Residual stress modeling and deformation measurement in laser metal deposition process. New Challenges in Residual Stress Measurements and Evaluation, 18/12/2019. https://doi.org/10.5772/intechopen.90539

[4] Y.R. Choi, S.D. Sun, Q. Liu, M. Bradt \& M. Qian, Influence of deposition strategy on the microstructure and fatigue properties of laser metal deposited Ti-6Al-4V powder no Ti-6Al-4V substrate. International Journal of Fatigue 130 (2020) 105236. https://doi.org/10.1016/j.ijfatigue.2019.105236

[5] H. Ali, H. Ghadbeigi \& K. Mumtaz, Effect of scanning strategies on residual stress and mechanical properties of selective laser melted Ti6A14V, Materials Science \& Engineering A, 712 (2018) 175-187. https://doi.org/10.1016/j.msea.2017.11.103

[6] I. Yadroitsev \& I. Yadroitsava, Evaluation of residual stress in stainless steel 316L and Ti6Al4V samples produced by selective laser melting. Virtual and Physical Prototyping, 10:2 (2015) 67-76. https://doi.org/10.1080/17452759.2015.1026045

[7] M. Turski \& L. Edwards, Residual stress measurement of a 316L stainless steel bead-on-plate specimen utilising the contour method. International Journal of Pressure Vessels and Piping, 86:1 (2009) 126-131. https://doi.org/10.1016/j.ijpvp.2008.11.020

[8] All Grating Fibres (AGF), 2020, FBGS, viewed $9^{\text {th }}$ October 2020

[9] Zhou, DP, Chen L \& Bao X, Distributed dynamic strain measurement using opticalfrequency domain reflectometry, Applied Optics, 55:24 (2016) 6735-6739.

https://doi.org/10.1364/AO.55.006735 